\title{
Prepare and prevent rather than repair and repent: Study of maternal mortality in tertiary care hospital
}

\section{Anjali Mundkur, Lavanya Rai}

Department of Obstetrics and Gynecology, Kasturba Medical College, Manipal University, Manipal, Karnataka, India

Address for the Correspondence: Dr. Anjali Mundkur, Department of Obstetrics and Gynecology, Kasturba Medical College, Manipal, Karnataka, India.

E-mail: dranjalisuneel@yahoo.in

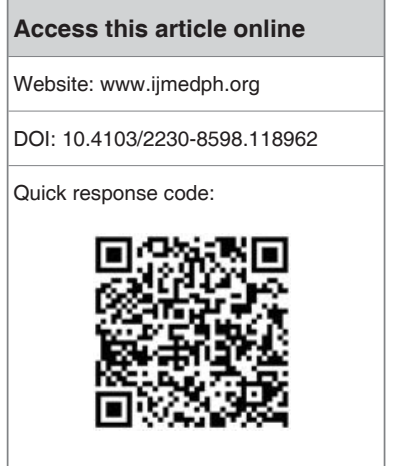

Context: Maternal death signifies the quality of healthcare provided in the population. It is the young, relatively healthy women who die of various reasons. Audit of such mortality would prevent the recurrence by taking appropriate measures. Aims: To find the causes of maternal mortality. Settings and Design: Retrospective observational study. Materials and Methods: All maternal deaths in a tertiary care referral center from January 2007 to September 2012 were studied for their demographic profile and causes of death. Results: All 62 women were referred from other healthcare units. Twenty-nine patients died within $24 \mathrm{~h}$ of admission and 33 women died after $24 \mathrm{~h}$ of admission. Death of 34 patients was due to direct obstetric causes and of 26 patients due to indirect obstetric causes. There were 2 maternal deaths due to accidental causes. Conclusions: The corrective action to prevent the recurrence of such deaths should be taken. Sepsis was found to be the commonest cause for maternal mortality followed by hemorrhage. It important to note that, in the present study, all mothers received antenatal care, had hospital delivery (none had home delivery), no teenage pregnancy or grand multigravidas, and no obstructed labor or rupture uterus, and yet they died. There is a change in the trend of causes of maternal mortality. Strengthening of the first referral units with equipment, blood bank, and adequately competent staff should be of prime importance. Continued medical education of the medial personnel at the periphery is required. Maternal deaths occur in inspite of atenatal care and hospital delivery which is alarming. Contributing factors may be delay in referral or the travel which should be looked in to inorder to minimize such death of young women.

Key words: Direct obstetric cause, indirect obstetric cause, maternal death, maternal mortality rate

\section{INTRODUCTION}

Three years remain until the 2015 deadline to achieve the Millennium Development Goals (MDG) adopted at the 2000 Millennium Summit. There are two targets for assessing progress in improving maternal health (MDG 5): Reducing the maternal mortality ratio (MMR) by three quarters between 1990 and 2015 and achieving universal access to reproductive health by 2015. Closer examination of maternal mortality level is needed to inform planning of reproductive health programs, to guide advocacy efforts and research at the national and international levels, and to inform decision-making. The audit for the reasons for maternal mortality in a resource poor country is extremely helpful in not only identifying the reasons but also in identifying the preventable causes of maternal mortality. The present study was undertaken to identify the preventable causes of maternal mortality in a tertiary care referral hospital.

\section{Maternal death}

International Statistical Classification of Diseases and Related Health Problems, Tenth Revision, 1992 (ICD-10), WHO defines maternal death as death of a woman while pregnant or within 42 days of termination of pregnancy, irrespective of the site and duration of pregnancy, from any cause related to or aggravated by pregnancy or its management but not from accidental or incidental causes. 


\section{AIMS AND OBJECTIVES}

To study the causes for maternal mortality in a tertiary care referral hospital.

\section{MATERIALS AND METHODS}

\section{Type of study}

Retrospective, observational.

\section{Period of study}

From January 1, 2007 to September 30, 2012. Records of all maternal deaths that were registered in the Department of Obstetrics and Gynaecology in tertiary care referral between January 1, 2007 and September 30, 2012 were included in the present study. They were studied for the immediate causes of death and the predisposing factors. The causes were identified as direct and indirect causes. Direct obstetric deaths are those resulting from obstetric complications of the pregnant state (pregnancy, delivery, and postpartum), from interventions, omissions, incorrect treatment, or from a chain of events resulting from any of the above. Indirect obstetric deaths are those resulting from previous existing disease or diseases that developed during pregnancy, and which were not due to direct obstetric causes, rather aggravated by physiological effects of pregnancy.

\section{RESULTS}

The centre had a total of 18, 458 deliveries from January 2007 to September 2012. Total number of maternal deaths during this period was found to be 62 . The maternal mortality ratio was 335 per 1,00,000 live birth. The age of the patients ranged from 21 to 37 years (mean age: $25 \pm 1.5$ years). All patients were referred to the centre receiving antenatal care elsewhere. The antenatal management of these patients were not known [Table 1].

All 62 women were referred from other healthcare units. Twenty-nine patients died within $24 \mathrm{~h}$ of admission and 33 women died $24 \mathrm{~h}$ after admission. The cause of death in 34 patients was due to direct obstetric causes and in 26 cases due to indirect obstetric causes. There were 2 maternal deaths due to accidental causes. (Statistical analysis was not obtained as the numbers were very small) [Table 2].

There were 13 (20.96\%) deaths due to post-partum hemorrhage. All of them where receiving antenatal care elsewhere. Six patients had risk factors for peripartum haemoglobin (PPH)-severe preeclampsia in 2 patients, 1 patient had placenta previa with placenta accreta, 1 had abruption, and 2 had severe preeclampsia with abruption. Cesarean section was done in a patient with placenta previa and placenta accreta in a private hospital with no blood facility available. Intra-operative bleeding was noticed and the patient was shifted to this hospital in shock with an accompanying radiology technician. Antenatal referral, timely

\begin{tabular}{|c|c|c|}
\hline Demographic details of women & No. & Percentage \\
\hline \multicolumn{3}{|l|}{ Age } \\
\hline $21-30$ years & 49 & 79.03 \\
\hline $31-40$ years & 13 & 20.96 \\
\hline \multicolumn{3}{|l|}{ Parity index } \\
\hline Primigravida & 17 & 24.19 \\
\hline Second gravida & 13 & 17.74 \\
\hline Third gravida & 07 & 11.29 \\
\hline Fourth gravida & 01 & 01.61 \\
\hline \multicolumn{3}{|l|}{ Antenatal (Period of Gestation) } \\
\hline Upto 13 weeks & 05 & 08.06 \\
\hline $13-28$ weeks & 10 & 16.12 \\
\hline$>28$ weeks & 23 & 35.48 \\
\hline Postabortal & 01 & \\
\hline Postnatal & 23 & \\
\hline \multicolumn{3}{|l|}{ Delivery status } \\
\hline Delivered & 35 & \\
\hline Induced abortion & 03 & 04.83 \\
\hline undelivered & 22 & 32.45 \\
\hline Laparotomy (ectopic pregnancy) & 02 & \\
\hline \multicolumn{3}{|l|}{ Place of delivery $(n=35)$} \\
\hline Tertiary care center & 13 & 20.90 \\
\hline Private healthcare center & 18 & 20.03 \\
\hline Government health units & 04 & 06.45 \\
\hline \multicolumn{3}{|l|}{ Mode of delivery $(n=35)$} \\
\hline Vaginal delivery & 15 & 42.85 \\
\hline Cesarean section & 20 & 57.14 \\
\hline Elective cesarean & 06 & \\
\hline Emergency cesarean & 14 & \\
\hline
\end{tabular}

Table 2: Identified direct obstetric causes of death

\begin{tabular}{lc}
\hline Causes of death & $\boldsymbol{n = 3 4}$ \\
\hline Postpartum hemorrhage & 13 \\
Sepsis with multi-organ dysfunction & 11 \\
Preeclampsia, eclampsia, and HELLP syndrome & 02 \\
Acute fatty liver of pregnancy & 04 \\
Ectopic pregnancy & 02 \\
Suspected amniotic fluid embolism & 02 \\
\hline
\end{tabular}

hysterectomy and well-equipped transport vehicle with medical personnel accompanying could have saved this patient. In 7 patients, there were no identifiable risk factors for post-partum hemorrhage. All these patients were referred from places not more than $60 \mathrm{~km}$ with $90 \mathrm{~min}$ travel time on an average. They were transported in patient transport vehicles without having a basic life support and no medical personnel accompanying. The condition of these patients might have deteriorated during the travel. Probably, if these patients had been transported in adequately equipped and staffed ambulance, mortality could have been avoided.

Of the 13 patients who died of post-partum hemorrhage, 11 had delivered vaginally and 2 had cesarean section. Requirement of surgical intervention was observed in 7 patients referred with PPH. Two patients who were already hysterectomised had laparotomy 
and internal iliac ligation in the centre. Hysterectomy with internal iliac artery ligation was done in 2 patients. Only hysterectomy was done in 1 patient. Laparotomy was done in 2 patients for hemoperitonium. All 13 patients had duration of stay (DIC) on admission.

Blood and blood products were given for 24 patients and a maximum of 92 pints of blood products were transfused for 1 patient who ultimately died.

It is important to note that the patients with risk factors identified antenataly should be referred to a higher center for institutional care at delivery. Appropriate risk categorization should be done by all antenatal care givers in order to identify and refer these women to centers with 24-h blood bank facility and multidisciplinary care.

In the era of ultrasonography, it is disheartening to note that 2 patients died of hemorrhagic shock due to ectopic pregnancy. One patient reached the hospital in irreversible shock. She was referred from a private hospital, where facility for laparotomy was available. Patient travelled $6 \mathrm{~h}$ in a state of shock. The crucial decision on laparotomy was delayed in this patient, which could have saved her. The second patient was referred to the center as anemia and laparotomy was done, but the patient died due to irreversible shock. Ectopic pregnancy should be suspected in all women in reproductive age group with anemia, pain abdomen, and irregular bleeding, who get admitted irrespective of the diagnosis.

In the present study, there were 2 patients who died of HELLP syndrome. Of which, 1 patient with pulmonary embolism was admitted to ICU in a serious condition and the second patient with DIC, who died of hypovolemic shock, was referred after a cesarean section done elsewhere.

There were 4 deaths due to acute fatty liver of pregnancy among the 7 patients admitted with the same condition. All were referred antenataly with jaundice. Pregnancy was terminated by cesarean in 2 patients due to obstetric indication. Intrauterine fetal death had occurred in 2 patients and pregnancy was terminated by vaginal delivery. Out of the 4 patients with acute fatty liver of pregnancy, 1 patient had postpartum hemorrhage due to DIC and multiorgan dysfunction, the remaining 3 patients had hepatic encephalopathy. This could be attributed to the disease. Studies have shown that there is $1-20 \%$ maternal mortality with acute fatty liver of pregnancy. ${ }^{[1]} \mathrm{It}$ is to be noted that a total of 7 cases of acute fatty liver of pregnancy was reported to the same institute during the study period, of which 3 survived [Table 3].

Infection-related mortality was the leading cause of death in the present study with 24 deaths $(37.09 \%)$. It was observed that there was a recent surge of viral pneumonia in the region. Viral pneumonia due to influenza H1N1 was the major cause for the mortality in these women. However, high index of suspicion and timely treatment with the antiviral treatment has drastically reduced the deaths due to $\mathrm{H} 1 \mathrm{~N} 1$ pneumonia.
Of the 11 patients with sepsis and multi-organ dysfunction, 4 had sepsis following cesarean section. Six patients were admitted with bacterial pneumonia. One of them was referred after vaginal delivery. One patient died of sepsis after gastroenteritis in early pregnancy. All these women were admitted to the intensive care unit (ICU) with multi-organ dysfunction. Infection in pregnancy should be treated aggressively to prevent such mortality.

Heart disease accounted for 8 maternal deaths. Congenital heart disease was present in 2 patients. Interstitial lung disease and Eisenmenger's syndrome contributed to the death. Peripartum cardiomyopathy was present in 1 patient, severe mitral stenosis in 2 patients, prosthetic heart valve in 2 patients, and severe pulmonary artery hypertension in 1 pateint. Three patients died after termination of pregnancy. Prosthetic heart valve was present in 2 patients and both of them died due to cardiac failure in early pregnancy. Pneumonia contributed for the death in 2 patients with severe mitral stenosis [Figure 1].

\section{DISCUSSION}

The maternal mortality ratio in India is 212 per 1,00,000 live births according to 2009 census, which is much above the objective of 109 per 1,00,000 live births as per the fifth Millennium Development Goal (MDG-5). ${ }^{[2]}$ Autopsy was not conducted in most of the women due to denial for the consent for the same by the bystanders. Hence, the cause of the death was attributed to the disease of presentation of these women. Reducing the maternal morbidity and mortality is the prime healthcare goal in developing countries. They are young healthy mothers who die majority of the times. Loss of a mother shatters the family. Studies have shown that infants who lost their mother during childbirth are more likely to die before reaching their second birthday than infants whose mother survives. ${ }^{[3]}$

In the present study, there were 62 maternal deaths out of 18,458 deliveries, presenting a maternal mortality ratio of 335 per

\begin{tabular}{lc}
$\begin{array}{l}\text { Table 3: Identified indirect obstetric causes of } \\
\text { death }\end{array}$ & $\mathbf{n = 2 6}$ \\
\hline Causes of death & 08 \\
\hline Heart disease & 08 \\
H1N1 pneumonia & 01 \\
Hepatic failure with HIV-positive status & 01 \\
Brain stem infarct & 01 \\
Hepatitis E with fulminant hepatic failure & 02 \\
Dengue shock syndrome & 01 \\
Malaria & 03 \\
Cortical vein thrombosis & 01 \\
Placental site trophoblastic tumor &
\end{tabular}

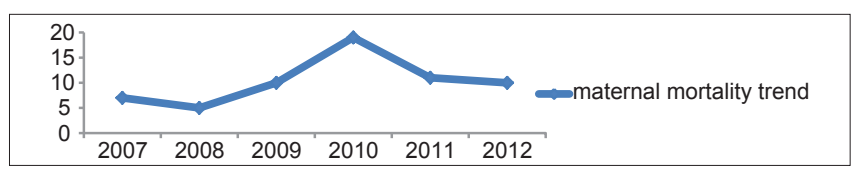

Figure 1: Maternal mortality trend 
1,00,000 live births. This high maternal mortality can be attributed to the fact that the study was conducted in the tertiary care referral center. Studies conducted in various tertiary care centres have reported MMR of 200 to 880 per 1,00,000 live births. ${ }^{[4,5]}$ Institutional mortality rates are 2-10 times higher as compared with field surveys, because most of the seriously ill patients were referred to the tertiary care centers. ${ }^{[6]}$

It is important to note that there were no mortality in women aged $<20$ years in the present study. Taneja showed that $78 \%$ of maternal deaths were observed in 20-30 years. ${ }^{[7]}$ There was no mother in the teens in the present study. Teenage pregnancy is rare in our district due to the high literacy among women.

Bera et al., revealed that, among the direct causes, hemorrhage was responbile for $23.8 \%$ deaths and sepsis for $16.4 \%$ deaths, and, among the indirect causes, jaundice was responsible for $9.9 \%$ deaths, followed by anemia and heart disease with $5.9 \%$ and $3.4 \%$ deaths, respectively. Sengupta et al., also noticed that, among the direct causes, hemorrhage $(12.40 \%)$ and sepsis $(17.82 \%)$ were the leading and, among the indirect causes, it was hepatitis (29.93\%) followed by anemia $(17.82 \%) .{ }^{[8]}$

In the present study, most of the women who died of infection had no contributing factor for the same. There was 1 patient with HIV-positive status. All 24 women were relatively healthy women who succumbed to death due to infection. These could have been due to delay in seeking medical attention or delayed referral, both of which could have been prevented. H1N1 infection symptoms are subtle to start with, but they progress rapidly to acute respiratory distress syndrome (ARDS). Educating the pregnant women regarding the need for medical care early during such illness and awareness of peripheral healthcare givers for early referral can reduce such mortality. The delay in referral to the tertiary care centre could also be due poor knowledge about medical disorders that complicate pregnancy among the peripheral health centre doctors. ${ }^{[9]}$

Most of the women who died of postpartum hemorrhage were admitted to the hospital in a state of disseminated intravascular coagulation. They were admitted in hypovolemic shock. Had these patients reached before this irreversible condition, they could have been saved. Studies have shown that two-thirds of the women who die of PPH do not have risk factors, hence all women should be considered at risk, and anticipation of postpartum hemorrhage should be done. ${ }^{[2]}$

Government should also make stringent rules and standards. License should be issued only to those healthcare units that meet the standards to manage such high-risk pregnancies. Moreover, government healthcare units should be made stronger by providing more facilities. All district hospitals should be strengthened with 24-h blood bank, better operation theatre facilities with adequate staffing, and basic life support (BLS)-equipped and manned ambulances.

Janani Suraksha Yojana scheme implemented by the government has reduced the home delivery rates with no significant reduction in the maternal mortality. ${ }^{[10]}$
Government ambulance (108) is neither being utilized by the people nor by the referring doctors. None of the patients were referred to the center in these ambulances. Unless the appropriate transfer is emphasized by the government, maternal mortality is unlikely to reduce. Legislation should be made to inform the district health officer regarding the transfer so that it can be monitored.

\section{CONCLUSIONS}

A systematic audit of each and every maternal death should be conducted for the analysis of root cause. The corrective action to prevent the recurrence of such deaths should be taken. In the present study, $70 \%$ of the maternal deaths were preventable. Sepsis was found to be the commonest cause for maternal mortality, followed by hemorrhage.

It important to note that, in the present study, all mothers had received antenatal care, had hospital delivery (none had home delivery), had no teenage pregnancy or grand multigravids, had no obstructed labor or rupture uterus, and yet they died. There is a change in the trend of causes of maternal mortality.

Strengthening of the first referral units with equipment, blood bank, and adequately competent staff should be of prime importance. Continued medical education of the medical personnel at the periphery is required.

\section{Suggestion}

Appropriate transfer with facilities for resuscitation and medical personnel accompanying the patient could prevent such mortality. Such medical personnel should be trained in the basic and advanced life support.

\section{ACKNOWLEDGMENTS}

Dr. Pratap Kumar N, Dr Muralidhar V Pai, and Dr Jyoti Shetty from the Department of OBG, Kasturba Medical College, Manipal, Karnataka, India.

\section{REFERENCES}

1. Joshi D, James A, Quaglia A, Westbrook RH, Heneghan MA. Liver disease in pregnancy. Lancet 2010;375:594-605.

2. Kumar S. Reducing maternal mortality in India: Policy, equity, and quality issues. Indian J Public Health 2010;54:57-64.

3. Tayade S, Bagde M, Shivkumar PV, Tayade A, Bagde N. Maternal death review to know the determinants of maternal mortality in a district hospital of central India. Int J Biol Med Res 2012;3:157-63.

4. Verma A, Minhas S, Sood A. A study of maternal mortality. J Obstet Gynaecol India 2008;58:226-9.

5. Jadhav A, Rote P. Maternal mortality changing trends. J Obstet Gynaecol India 2007;57:398-400.

6. Kaur D, Kaur A. A retrospective study of maternal mortality in government medical college. J Obstet Gynaecol Family Welfare1999;5:18-22.

7. Taneja PV. Maternal mortality in Madhya Pradesh. J Obstet Gynaecol India 2000;50:59-61.

8. Bangal VB, Purushottam A. Giri B, Garg RC. Maternal mortality at a tertiary care teaching hospital of rural India: A Retrospective Study. Int J Biol Med Res 2011;2:1043-1. 
9. Iyer A. Verbal autopsies of maternal deaths in Koppal, Karnataka: Lessons from the grave. BMC Proc 2012;6(Suppl 1):P2. Available from: http://www. biomedcentral.com/1753-6561/6/S1/P2 [Last accessed 2012 Jan 16].

10. Guin G, Sahu B, Khare S, Kavishwar A. Trends in maternal mortality and impact of Janani Suraksha Yojana on Maternal mortality ratio in a tertiary. J Obstet Gynaecol India 2012;62:307-11.
How to cite this article: Mundkur A, Rai L. Prepare and prevent rather than repair and repent: Study of maternal mortality in tertiary care hospital. Int J Med Public Health 2013;3:163-7.

Source of Support: Nil, Conflict of Interest: None declared. 